\title{
Impact of Simultaneous Movements on the Perception of Safety, Workload and Task Difficulty in a Multiple Remote Tower Environment
}

\author{
Maria Hagl \\ German Aerospace Center \\ Lilienthalplatz 7 \\ 38108 Braunschweig \\ $+495312952916$ \\ Maria.Hagl@dlr.de
}

\author{
Maik Friedrich \\ German Aerospace Center \\ Lilienthalplatz 7 \\ 38108 Braunschweig \\ $+495312952598$ \\ Maik.Friedrich@dlr.de
}

\author{
Joern Jakobi \\ German Aerospace Center \\ Lilienthalplatz 7 \\ 38108 Braunschweig \\ $+495312952536$ \\ Joern.Jakobi@dlr.de
}

\author{
Sebastian Schier-Morgenthal \\ German Aerospace Center \\ Lilienthalplatz 7 \\ 38108 Braunschweig \\ $+495312952337$ \\ Sebastian.Schier@dlr.de
}

\begin{abstract}
Providing air traffic service to more than one aerodrome is a key concept within Remote Tower. So-called Multiple Remote Tower Operations (MRTO) are expected to be more cost-efficient and user-friendly. On the one hand, their anticipated benefit is to maintain smaller airports that are currently non-profitable due to low traffic numbers, high staffand tower maintenance costs. On the other hand, MTRO offer equally distributed and constant activity for air traffic controllers (ATCOs), with the expectation to lower risks of human error due to boredom or sleepiness at work. However, multiple tasking challenges arise if one ATCO needs to handle traffic at three airports simultaneously. Thus, combinations of visual, audio, vocal and haptic tasks need to be performed for more than just one location. Therefore, this paper addresses the impact of simultaneous movements on perceived safety, workload and task difficulty. Descriptive results show that with the increase of simultaneous movements, providing ATC is perceived as being more efficiency-critical, more demanding in workload and task difficulty increases as well. It was not tested if the differences were significant, since statistical conditions haven't been met. Results show that no situation containing simultaneous movements was perceived as a threat to safety, good workload or the ability to provide ATC. The discussion shows why the impact of simultaneous movements might not only affect MRTO but also single remote or conventional tower environments.
\end{abstract}

\section{TABLE OF CONTENTS}

1. INTRODUCTION..................................................... 1

2. METHOD................................................................ 3

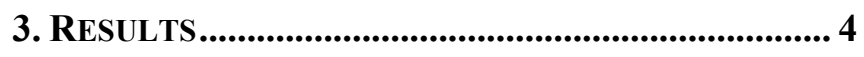

Christopher Stockdale

German Aerospace Center

Lilienthalplatz 7

38108 Braunschweig

$+17248846765$

Christopher.Stockdale@dlr.de
4. DISCUSSION ......................................................................6

ACKNOWLEDGEMENTS ............................................... 7

REFERENCES ........................................................... 7

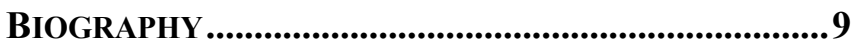

\section{INTRODUCTION}

In 2016, passengers have flown around the world more than ever before [1]. Also, more freight than in the previous years has been carried world-wide [2]. But while the tendency to transport more travelers and goods by air doesn't seem to cease [3], it appears that only mid-to-large sized airports profit from higher passenger numbers and cargo transport. Regional and small sized airports however become fairly unprofitable around Europe [4] [5] and even need to be closed down [6] because of their financial burden. But not only financial deficits are a danger for the concerned regional and small sized airports. Air traffic controllers (ATCOs) at these airports suffer from low workload by having to deal with the small amount of traffic. Newspapers even reported on ATCOs that fell asleep being alone on shift without constant activity [7]. These cases are only reported because, after a long period of low workload, the ATCOs had to be vigilant and reactive all the sudden and couldn't meet adequately meet their duties. As a matter of fact, operators are more susceptible to commit errors under low workload conditions, especially when they are tired [8] [9]. To outbalance high workload fluctuation within ATCOs and to make regional airports profitable again, the German Aerospace Center (Ger. Deutsches Zentrum für 
Luft- und Raumfahrt e.V.; $A b b$. DLR) in Braunschweig developed "Remote Tower" in 2002. The concept consists of replacing a conventional tower with a remote tower. This remote tower, based anywhere, is comprised of a set of displays portraying the tower's controls as well as a reconstructed virtual "out-of-the-window" view of the airfield [10]. An example of the aforementioned Remote Tower system went operational in 2015 at Örnsköldvik airport in Sweden. Thus, air traffic occurring in Örnsköldvik is able to be observed and controlled from a Remote Tower Center (RTC) located in Sundvall [11].

A logical next step is the creation of a workplace that allows ATCOs to control more than one airport from a single, dedicated, RTC. This concept does not only permit a more economically-efficient system by circumventing the costly construction and maintenance of conventional tower facilities, it is also an effective method of reducing ATCO boredom and sleepiness by keeping workload more constant. However, a serious challenge is susceptible to arise in the case of a single ATCO managing the surveillance of several airports singlehandedly. We have dubbed such a situation: Inter-Multitasking. Before explaining what we mean by this neologism, we will give a definition of Multitasking and shortly describe its challenges, followed by describing Multitasking in a current air traffic control environment. While it seems to be difficult to agree on an absolute or widely understood definition of Multitasking, most authors conclude that it implies simultaneous task handling, quick task switching [12] or attempting to manage a rapid succession of tasks [13]. From past studies, factors such as task difficulty, training, task combination or task similarity appear to influence the efficiency of Multitasking and its effects on performance and security. Concerning task difficulty, [14] argues that simple tasks need less attention than difficult tasks and can therefore be executed much easier in parallel. He further states that untrained processes need more attention than trained processes which can have an adverse effect on efficient Multitasking. [15] explain that processes can be automatized by training and facilitating Multitasking strategies [16]. Thus, a recall of registered key elements related to a past situation or the direct access to it supports the efficiency of Multitasking [17]. However, it shouldn't be neglected that even well-trained "experts" are accident prone when it comes to Multitasking [18]. Next to task difficulty and training, [19] argue that the performance of Multitasking depends on the task combination. For instance, if one is attempting to write while simultaneously listening to an unrelated conversation, the individual in question will experience a form of mental interference. [20] conclude that these interferences are prone to appear when dealing with similar tasks. It might therefore be more difficult to execute two visual tasks at the same time than simultaneously dealing with aural and visual tasks. Wickens' Multiple Resource Theory [21] and the Integrated Theory of Concurring Multitasking by [22] resume challenging factors and their interaction in completer models.
In current air traffic control (ATC), the ability to multitask is a required criterion when selecting ATCOs [23], since it's needed in their everyday work, especially in situation with increased workload. In a conventional or single remote tower, the ATCO's multitasking strategies are restricted to task combinations of a visual, aural, vocal and haptic nature in a single aerodrome. Challenges arise when it comes to Multiple Remote Tower Operations (MRTO), since these task combinations are not only restricted to one aerodrome. So-called "Inter-Multitasking strategies" are therefore needed to respond to the complexity that can arise with each additional aerodrome. To create successful InterMultitasking strategies, ergonomic strategies have to be established to secure operation, enable adequate performance, and cost-efficient use of MRTO systems at first. Within the European SESAR project "Remote Tower for Multiple Airports" ${ }^{\text {c }}$ current challenges of MRTO are evaluated. Key parameters such as time of the day, traffic volume, traffic complexity, traffic distribution, operational modes, runway conditions, wind conditions and visibility conditions have been proposed as conditions that interfere with Safety and Human Performance in MRTO [24]. Therefore, in collaboration with the Lithuanian air navigation service provider (ANSP) Se Oro Navigacija and Frequentis, an industry partner specialized on securitycritical information- and communication systems, DLR conducted a study to evaluate the influence of Traffic Volume and Traffic Complexity on Human Performance and Safety in a real-time simulation environment [24]. In the same context and with a more specific focus on Multitasking, DLR explored the impact of number of Simultaneous Movements on the ATCO's workload, operational safety and task difficulty. By simultaneous movements, we understand the number of simultaneous movements on the frequency. ATCOs had to deal with simultaneous movements between two up to six at a time. Taking into account the preexisting literature, the following hypotheses are proposed:

H1,1: By increasing the number of simultaneous movements, the handling of ATC will be perceived as more safety-critical.

H1,2: By increasing the number of simultaneous movements, the ATCO's workload will increase as well.

H1,3: The ATCO's workload will be higher when dealing with similar simultaneous movements than when dealing with different simultaneous movements.

H1,4: By increasing the number of aerodromes from two to three, the handling of ATC relevant tasks will be perceived as more difficult.

\footnotetext{
${ }^{1}$ Project funded by SESAR Joint Undertaking under the European Union's Horizon 2020 research and innovation program under grant agreement No 730195 .
} 
In the following, the method to conduct this explorative study is explained in terms of participants, material and procedure. In the result section, findings will be detailed. Results will be explained and analyzed in the discussion part.

\section{METHOD}

\section{Participants}

6 Lithuanian ATCOs (all male) participated in the study from March $19^{\text {th }}$ to March $27^{\text {th }} ， 2018$. They were aged between 25 and 36 years $(M=29.33 ; N=6 ; S D=3.73)$ and had been working in Approach and Tower Control for 3.73 years $(M=3.73 ; N=6 ; S D=2.43 ; M I N=1 ; M A X=8)$ on average. Five out of six ATCOs had been familiar with Remote Tower concepts before participating in the study but none of them had ever participated in a related study or operated a Remote Tower system.

\section{Material}

To render the MRTO test environment as realistic as possible, DLR designed a prototype around a Narsim realtime simulation platform (cf. Figure 1). It was extended by an electronic smart-strip planning tool developed by Frequentis. The out-of-the-window-view of two small sized and one medium sized airport were displayed on a $208^{\circ}$ horizontal and $32^{\circ}$ vertical video panorama, extended by a $52^{\circ}$ horizontal and $32^{\circ}$ vertical pan-tilt-zoom camera on the right side.

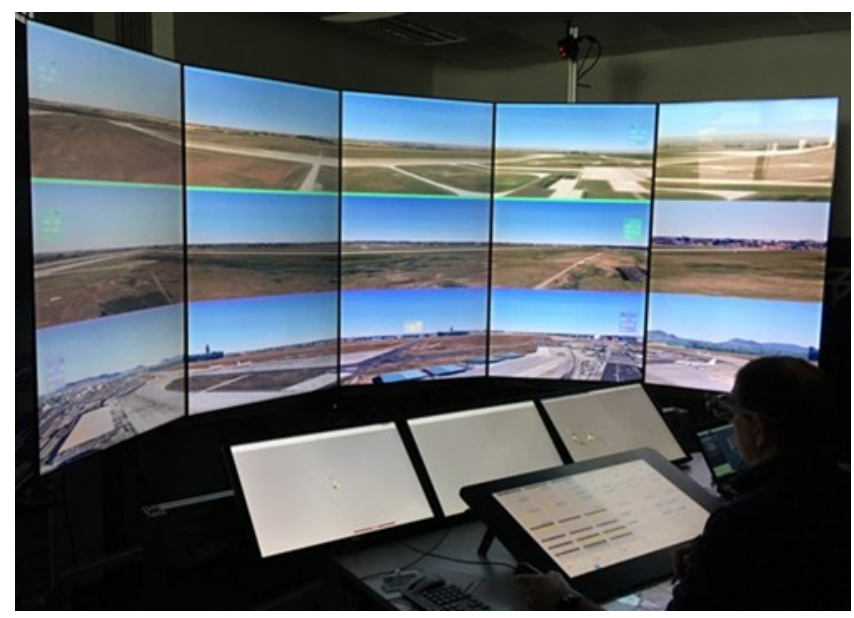

Figure 1. MRTO Real-Time Simulation Platform with extended Smart-Strip Planning Tool

For a more ergonomic head position throughout the study, the order of aerodromes was presented from the least frequented aerodrome on top to the highest frequented aerodrome on the bottom section. On the electronic planning tool, this order was presented from left to right. A better distinction of the aerodromes was visually enabled by the means of a color code. Each aerodrome was assigned a specific color in the video panorama which was the same as on the electronic flight strip system. For voice communication, ATCOs were provided a headset and microphone. All frequencies (ground- and air) were coupled and the audio output occurred via speakers. When the ATCO was talking on the radio, each color bar blinked. When a pilot called in, only the color bar of the concerning aerodrome blinked. More overlaid information (cf. Figure 2) were integrated in the video panorama, such as additional weather information and runway designators. Aircraft- and vehicle labels including call sign, aircraft type, flight phase, altitude and speed were presented to enable a maximal headup driven work experience [25].
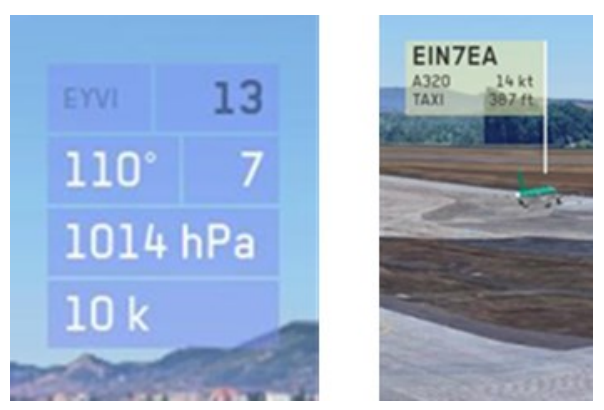

Figure 2. Overlaid Weather Information (left) and Aircraft Label Information (right)

For the simulation content, four scenarios à 50 minutes were implemented by DLR. Constant feedback from ON allowed to provide a realistic, but challenging traffic situation for the collaborating ATCOs. For a solid methodological framework, several parameters were settled fix in each scenario over all three aerodromes. Traffic was therefore distributed unevenly $($ Top $=23 \%$; Middle $=32 \%$; Bottom $=$ $45 \%$ ). The operational modes were fixed to normal, runway tarmac conditions were all good, and it was daytime at all three aerodromes. Visibility conditions were all VMC with CAVOK no clouds and wind conditions were all calm. In a pretest, the traffic volume, corresponding to 30 movements per hour, was judged too high. To better meet the ANSP's work conditions without neglecting the occurrence of simultaneous movements, the traffic volume was set to either 24 air movements per hour (Scenario 1 and 2) or fixed to 24 air- plus 2 ground vehicle movements per hour (Scenario 3 and 4). The proportion of Instrumental Flight Rules (IFR) and Visual Flight Rules (VFR) were either 90\% to $10 \%$ (Scenario 1 and 3 ) or $80 \%$ to $20 \%$ (Scenario 2 and 4). Furthermore, the scenarios were created to provoke simultaneous departures, simultaneous landings (similar simultaneous movements) and simultaneous departure and landings (different simultaneous movements). In all scenarios, ATCOs had to deal with two up to six simultaneous movements at a time. By the means of these four scenarios, we aimed at testing the impact of the number of simultaneous movements on the Expert Observer's safety evaluation, the ATCO's workload and perceived task difficulty. 


\section{Procedure}

ATCOs participated pair-wise in the study but performed alternately in different roles over two simulation days. When arriving, both ATCOs were briefed together about the study's objectives, study material and procedures. To get familiar with the system and working procedures, they performed a training session of 30-40 minutes each, depending on their personal evaluation of readiness for the first simulation run. While one ATCO was controlling actively three aerodromes from a single remote tower working position, the other one was completing a CooperHarper-Scale [26] adapted by DLR, to evaluate safety and to ask the active ATCO's workload at specific situations. These specific situations included the number of simultaneous movements, the implied aerodromes, similar simultaneous movements (departures or landings) and different simultaneous movements (departures and landings). Instantly, the Expert Observer could rate if the situation could be solved without major impairment $(1=n o$ impairment to 3 = minor impairment) if the situation could be solved by measures of capacity $(4=$ minor impairment of efficiency to $6=$ high impairment of efficiency) or if the situation could be solved by the means of reducing safety ( 7 $=$ impairments in prediction of traffic development to $10=$ major impairment of safety). At the same instant, the active ATCO was asked to rate his current workload on an ISAworkload scale [27]. The ATCO could indicate his level of workload from $(1=$ underutilized, $2=$ relaxed, $3=$ comfortable busy place, $4=$ high, $5=$ excessive). Their roles changed after each scenario. To mitigate potential learning effects, the order of presented scenarios was randomized. After each simulation run, the active ATCO completed a post-run questionnaire providing rating his experience of task difficulty while having to deal with simultaneous movements on a 7-point decision scale $(0=$ very easy to $6=$ very difficult). After having competed four scenarios each, the ATCOs filled out a post-exercise questionnaire before giving feedback in a debriefing phase.

\section{RESULTS}

Data were collected by quantitative approach using questionnaires (post-run and post-exercise) and adapted scales (within-run) as well as by qualitative approach applying semi-directive interview methods (post-exercise). This section addresses the descriptive results concerning the impact of Simultaneous Movements on the perception of Safety Evaluation, Workload and Task Difficulty. It further presents the qualitative feedback we obtained by the ATCOs on MRTO procedures and the used system from an HMI perspective.

Impacts of the number of Simultaneous Movements on the Safety Evaluation by the Expert Observer

It was postulated that the handling of ATC will be perceived as more safety-critical with increasing number of simultaneous movements. In the descriptive statistics, this assumption can be confirmed (cf. Figure 3). By passing from two simultaneous movements $M=1.31 ; N=13 ; S D=$ $0.46 ; \operatorname{Min}=1 ; \operatorname{Max}=2$ to three $M=1.74 ; N=31 ; S D=$ $0.67 ; \operatorname{Min}=1 ; \operatorname{Max}=4$, four $M=2.17 ; N=47 ; S D=0.63$; $\operatorname{Min}=1 ; \operatorname{Max}=4$, and five movements $M=2.61 ; N=23$; $S D=0.82 ; \operatorname{Min}=1 ; \operatorname{Max}=4$, the safety-critical score increases. At six movements, the safety-critical score slightly drops to $M=2.33 ; N=6 ; S D=0.75 ; \operatorname{Min}=2 ; \operatorname{Max}$ $=4$. There is a positive correlation between the number of simultaneous movements and the safety-critical score $r=$ $0.47, p \leq 0.001$. Nevertheless, the mean safety-critical score always was between 1 and 3 which corresponds to judging the traffic situation with "no impairment of efficiency" to "minor impairment of efficiency". In $6.6 \%$ of the situations concerning either three, four, five or six simultaneous movements, ATC has been judged as being susceptible to leading to "unpleasant delays".

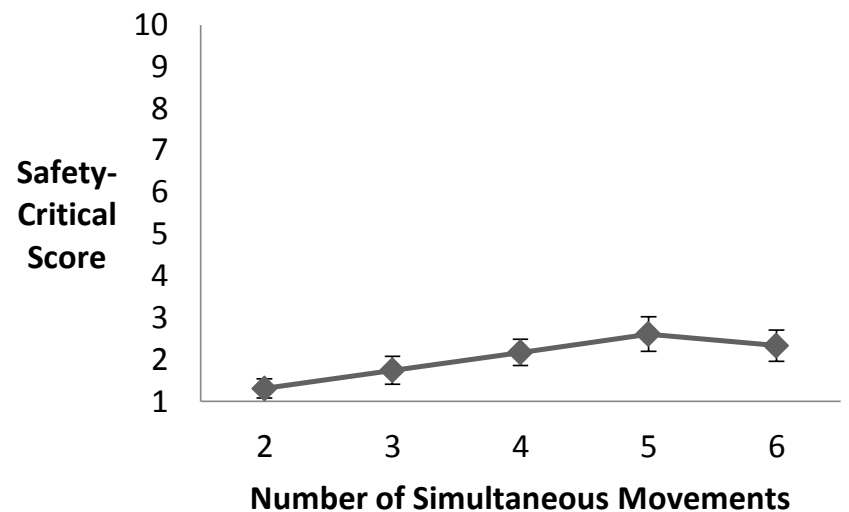

Figure 3. Safety-Critical Score per Simultaneous Aircraft Movements evaluated by Expert Observer

Impacts of the number of Simultaneous Movements on the perceived Workload by the operating ATCO

It was postulated that the perceived workload increases with increasing number of simultaneous movements. Regarding the descriptive statistics, as assumed, the ATCO's workload increases when passing from two simultaneous movements $M=1.54 ; N=13 ; S D=0.5 ; \operatorname{Min}=1 ; \operatorname{Max}=2$ to three $M=$ $1.84 ; N=31 ; S D=0.68 ; \operatorname{Min}=1 ; \operatorname{Max}=4$, four $M=2.38$; $N=47 ; S D=0.53 ; \operatorname{Min}=1 ; \operatorname{Max}=4$ and five movements $M=2.61 ; N=23 ; S D=0.49 ; \operatorname{Min}=2 ; \operatorname{Max}=3$. At six movements, the workload slightly drops to $M=2.5 ; N=6$; $S D=0.5 ; \operatorname{Min}=2 ; \operatorname{Max}=3$ (cf. Figure 4). There is a positive correlation between the number of simultaneous movements and the perceived workload $r=0.491, p \leq$ 0.001 . Nevertheless, the mean workload score always is between 1 and 3 which correspond to feeling either "underutilized", "relaxed" and "in comfortable busy place”. In debriefing [28], all ATCOs stated that their workload was fairly higher when they had to handle a greater amount of simultaneous movements. 


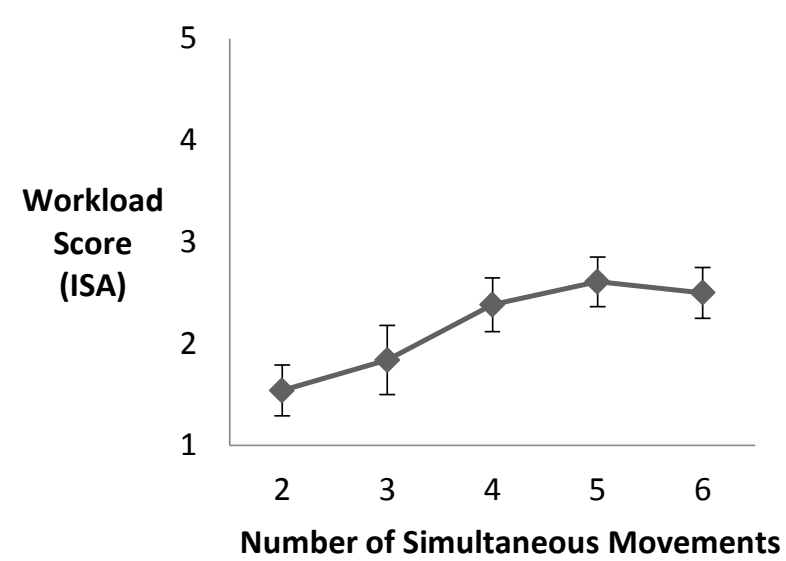

Figure 4. Workload Score (ISA) per Simultaneous Aircraft movement perceived by operating ATCO

Impacts of the number of Simultaneous Movements on the perceived Workload by the operating ATCO, depending on task similarity

It was postulated that the ATCO's workload increases when dealing with similar simultaneous movements compared to different simultaneous movements. Considering the results, no large difference could be identified between the perceived workload in case of dealing with similar simultaneous movements compared to different simultaneous movements. In fact, at two simultaneous aircraft movements, the perceived workload is $M=1.5 ; N=$ $10 ; S D=0.5$ not that much lower than at $M=1.57 ; N=3$; $S D=0.47$. The same applies to four simultaneous movements $M=2.3 ; N=10 ; S D=0.64$ compared to $M=$ $2.41 ; N=37 ; S D=0.49$. At three similar simultaneous movements $M=1.57 ; N=14 ; S D=0.49$, the workload seems to be lower than at three different simultaneous movements $M=2.06 ; N=17 ; S D=0.72$. At five simultaneous movements, only one case of similar simultaneous movements has appears $M=2 ; N=1 ; S D=0$. It is therefore not useful to compare means with five different movements $M=2.64 ; N=22 ; S D=0.48$. In debriefing [28], ATCOs estimated that it was more likely to confuse inbound traffic at several aerodromes than inboundand outbound traffic, especially when having to deal with similar call signs and taxiways. They suggested an adaptation of phraseology to avoid confusion related to simultaneous movements and task similarity.

Impacts of the number of Simultaneous Movements on the perceived Task Difficulty comparing two aerodromes to three aerodromes

It was postulated that the handling of ATC will be perceived as more difficult with increasing number of aerodromes. In descriptive statistics, this assumption can be confirmed (cf. Figure 5). Perceived difficulty arises when providing ATC to simultaneous outbound movements at two aerodromes $M$
$=0.96 ; N=24 ; S D=0.79 ; \operatorname{Min}=0 ; \operatorname{Max}=3$ compared to three aerodromes $M=1.57 ; N=21 ; S D=1.0 ;$ Min $=0 ;$ Max $=3$. This tendency can also be observed for simultaneous inbound movements at two aerodromes $M=1.29 ; N=24$; $S D=1.06 ; \operatorname{Min}=0 ; \operatorname{Max}=4$ compared to three aerodromes $M=1.95 ; N=20 ; S D=1.02 ; \operatorname{Min}=0 ; \operatorname{Max}=4$ or simultaneous inbound and outbound movements at two aerodromes $M=1.92 ; N=24 ; S D=0.95 ; \operatorname{Min}=1 ; \operatorname{Max}=4$ compared to three aerodromes $M=2.95 ; N=22 ; S D=0.84$; $\operatorname{Min}=1 ; \operatorname{Max}=4$. It can be stated that none of the above mentioned ATC relevant tasks was perceived as "rather difficult", "difficult" or "very difficult" on average. There was a strong positive correlation between the number of aerodromes and the perceived task difficulty to handle simultaneous outbound movements $r=0.876, p \leq 0.001$, simultaneous inbound movements $r=0.905, p \leq 0.001$ and simultaneous traffic mix $r=0$ 0.894, $p \leq 0.001$. These results show the same tendency when taking into account the order of runs. Considering Figure 5, it appears that perceived task difficulty seemed to be lower when having to deal with similar simultaneous movements than with simultaneous mixed movements.

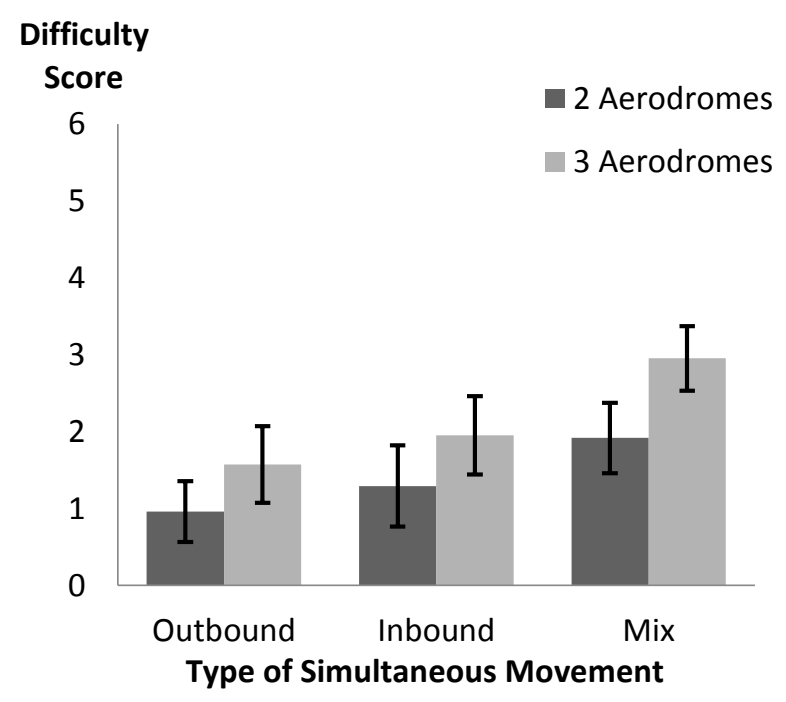

Figure 5. Comparison Perceived Task difficulty at 2 vs. 3 Aerodromes Outbound, Inbound, Mix

In debriefing [28], ATCOs stated not feeling comfortable dealing alone with this traffic amount- and composition at three aerodromes in real life. ATCOs further suggested that providing ATC to two or three aerodromes simultaneously could be possible with adapted working conditions (e.g. lower traffic volume, adapted traffic composition), training and supervisor support They also agreed that MTRO could be a sustainable concept for the future under strictly defined procedures and only for distant and rarely used airports. 


\section{DISCUSSION}

As statistical conditions have not been met to conduct inferential statistical analysis, results were reported descriptively only. Even though participants of the study were all operational ATCOs and the sample therefore consisted of a homogeneous expert sample, the significance of results has to be interpreted with caution and considered to serve explorative means, such as showing first tendencies of a bigger framework. In the following, the results of each tested hypothesis will be discussed separately before concluding with a general statement.

Impacts of the number of Simultaneous Movements on the Safety Evaluation by the Expert Observer

First it was assumed that handling of ATC will be perceived as more safety-critical with increasing number of simultaneous movements. Results support this hypothesis in the comparison of means. As a matter of fact, the safetycritical score augments constantly with the increase of simultaneous movements. Interestingly, the ATCOs workload slightly decreases at six simultaneous movements. Regarding the small amount of collected data at six simultaneous movements, it is doubtful that the difference between five and six simultaneous movements is significant. Moreover, it is likely that the perceived safety stagnates at a certain level of simultaneous movements or even constantly increases. The strong significant positive correlation found between the number of simultaneous movements and higher safety critical score support this idea. However, it is important to note that in average, situations with simultaneous movements have been judged as having no impact or minor impact of efficiency impairment. In only $6.6 \%$ of situations when dealing with either three, four, five or six simultaneous movements at a time, impairment of efficiency was detected and judged as potentially leading to unpleasant delays. This number is relatively low compared to $93.4 \%$ of the situations where no impairment of efficiency was detected. In summary, throughout the experiment, no safety-critical situations were detected. However, results suggest that with augmenting the number of simultaneous aircraft, the safety-critical score rises to impairment of efficiency. These results have to be taken into account while dealing with MRTO and need to be further investigated.

Impacts of the number of Simultaneous Movements on the perceived Workload by the operating ATCO

Concerning the assumption that the ATCO's workload will increase by augmenting the amount of simultaneous movements, results support this hypothesis. When passing from two simultaneous movements to three, four and five simultaneous movements, the ATCO's workload increases constantly at a similar degree. The difference of ascent seems to reach its peak between three and four movements. Interestingly, the ATCOs workload slightly decreases at six simultaneous movements. Regarding the small amount of collected data at six simultaneous movements, it is doubtful that the difference between five and six simultaneous movements is significant. Moreover, it is possible that the workload stagnates at a certain level of simultaneous movements or even increases constantly. The strong positive correlation found between the number of simultaneous movements and workload support this idea. It was interesting to find that even though the ATCO's average workload was rated as "underutilized", "relaxed" and never exceeded "comfortably busy place" on the ISAScale [27], the qualitative feedback revealed slightly different insights on workload. During debriefing, ATCOs stated that their workload was fairly higher when the number of simultaneous movements increased. An explanation for this refers to the interpretation of the ISAscale. Although being briefed about the meaning corresponding to each number on the scale and having the information in reach during the experiment, ATCOs didn't much consult the description when they gave their answers on workload. When comparing the signification of the average answers given and the qualitative feedback, it is possible that the ATCOs associated "1" with "workload $o k$ " and then established gradual references to the other numbers on the scale. Another explanation is that they were not willing to admit their current workload to their fellow ATCO who was in the role as an Expert Observer. In both cases, it is therefore not advisable to literally interpret the meaning of each number on the scale but to rather concentrate on the increase of workload when having to deal with more simultaneous movements which was not only found by comparison of means and correlation analysis but also confirmed orally in the debriefing session after the experiment [28]. In conclusion the number of simultaneous aircraft movements has to be taken into account not only for further MRTO working procedure adaptations but also for conventional towers. Of course, one cannot always exclude the occurrence of simultaneous traffic, neither when operating more than one aerodrome, nor when operating only one aerodrome from a conventional or single remote tower, but flight plans can be scheduled in a manner that simultaneous movements are likely to occur less often.

Impacts of the number of Simultaneous Movements on the perceived Workload by the operating ATCO, depending on task similarity

Referring to what is known about task interference [20], we assumed that workload increases when having to deal with similar simultaneous movements (e.g. simultaneous departures; simultaneous landings) compared to different simultaneous movements. Results show that the perceived workload doesn't seem to differ in similar conditions compared to different conditions. It even seems that similar movements induce slightly less workload than different movements. At three simultaneous movements, a greater difference appears. At five simultaneous movements the difference is even higher. However, this comparison should not be taken into account since we compared one value collected at five similar simultaneous movements to 22 
values at five different simultaneous movements. Moreover, by splitting the data into two different groups such as similar simultaneous movements and different simultaneous movements per several simultaneous aircraft movements, we lose more data than gaining valuable information. Next to this, the feedback gathered in debriefing shows that the comparison of means evaluating the impact of similarity of simultaneous movements on workload has to be interpreted with caution and certainly should not be overestimated.

Impacts of the number of Simultaneous Movements on the perceived Task Difficulty comparing two aerodromes to three aerodromes

Regarding the hypothesis that handling of ATC will be perceived as more difficult with increasing number of aerodromes, results support the hypothesis. Nevertheless it is important to note that in our setting, with increasing number of aerodromes the likelihood for more simultaneous movements increased as well. Results show that the perceived task difficulty arises when providing ATC to simultaneous outbound movements at three aerodromes instead of two aerodromes. The same applies for simultaneous inbound movements and simultaneous inbound- and outbound movements. The strong positive correlation between the number of aerodromes and perceived task difficulty also supports the hypotheses. These results are not surprising, given the fact that the participating ATCOs had no experience in handling ATC at two or three aerodromes simultaneously prior to the study. They therefore need more attention to complete tasks and each additional aerodrome with a higher probability for more even simultaneous movements might augment difficulty. Even though they got more and more familiar with the concept from their first to their last experimental run, they judged on average that it was easier providing ATC to two aerodromes than to three aerodromes. As ATCOs stated in debriefing, their workload was higher when having to deal with traffic at all three aerodromes simultaneously compared to two aerodromes, especially when VFR emerged. In this experimental setting, it is only logic that task difficulty and workload arise because it was more likely to have more simultaneous movements on the frequency when dealing with three aerodromes compared to two aerodromes. For future studies it would be interesting to test if the difference of perceived task difficulty at one, two or more aerodromes is significant with the same amount of simultaneous traffic in each condition. It is very likely that it is not the number of aerodromes that increases task difficulty and workload but, same as in conventional towers today, the number and type of simultaneous tasks to handle.

A surprising result appeared when analysing the perceived task difficulty for similar simultaneous movements (simultaneous inbound and simultaneous outbound) and for different simultaneous inbound- and outbound movements. It seems that similar movements are less difficult to handle than different movements. For future research, this observation should be tested further to evaluate if the difference in difficulty perception is significant. In general, it was interesting to observe that on average, the ATC tasks were perceived from "very easy" to "neither easy nor difficult". One explanation refers to the 7-point decision scale we chose for evaluating task difficulty. It could have been too sensible and there might have been too many answer possibilities at low gradual steps to choose. For future questionnaires, we recommend therefore a classical 5-point Likert scale.

In conclusion, the study aimed to test the impact of simultaneous movements on workload, safety and task difficulty in an explorative manner, based on previous findings of Multitasking. To make a maximum amount of simultaneous movements possible, a higher traffic load than ATCOs face usually was necessary. This amount of traffic is not realistic for future MRTO operations. It is important to note that we were not looking for setting a limit of simultaneous movements for one ATCO that operates three different aerodromes. It would be unwise and even dangerous to think that one could be able to determine such a "magic number". The same logic applies to determine the "correct" number of aerodromes that can be operated simultaneously. Safety, workload and task difficulty are highly dependent on other factors (e.g. traffic situation, visibility conditions, flight operation modes, etc.) that have been omitted in this real-time simulation due to limited resources. Simultaneous movements are only one piece within a bigger framework and can occur in conventional or single remote tower environments just as in MRT environments. We are very well aware of the fact that next to subjective questionnaire and briefing techniques, physiological measures [29] would be adequate to complete the results in a more objective manner. In the future, limits have to be explored and to be studied in their own specific and practical context with the concerned operators to give a better indication for what can be operated in a safe and efficient manner and how to design an adapted MRTO work environment. After all, the ATCOs agreed that MRTO could be a sustainable concept for the future but only under strictly defined procedures and adapted working conditions.

\section{ACKNOWLEDGEMENTS}

The authors thank SESAR Joint Undertaking for funding this project.

\section{REFERENCES}

[1] The World Bank (2018). Air transport, passengers carried. Retrieved from: https://data.worldbank.org/indicator/IS.AIR.PSGR

[2] The World Bank (2018). Air transport, registered carrier departures worldwide. Retrieved from: https://data.worldbank.org/indicator/IS.AIR.DPRT

[3] The Statistics Portal. Annual growth in global air traffic passenger demand from 2005 to 2018. Retrieved from: 
https:/www.statista.com/statistics/193533/growth-ofglobal-air-traffic-passenger-demand/

[4] Mathisen, T. A., \& Solvoll, G. (2012). Reconsidering the regional airport network in Norway. European Transport Research Review, 4(1), 39-46.

[5] Koschnick, W. J. (2015). Deutschland im regionalen Flughafen-Wahn. Retrieved from: https://www.heise.de/tp/features/Deutschland-imregionalen-Flughafen-Wahn-3376545.html?seite $=$ all

[6] Pidd, H. (2014). "A sad loss for Blackpool as unprofitable airport to close." Retrieved from: https://www.theguardian.com/uk-news/2014/oct/08/sadloss-holidaymakers-workers-blackpool-airport-closure

[7] Smith, G. (2011). Night shifts are "insane" says air traffic controller who fell asleep on the job. Retrieved from: http:/www.dailymail.co.uk/news/article-2050912/Nightshifts-insane-says-air-traffic-controller-fell-asleep-job.html

[8] Fisher, C. D. (1993). Boredom at work: A neglected concept. Human Relations, 46(3), 395-417.

[9] Weinger, M. B. (1999). Vigilance, boredom and sleepiness. Journal of Clinical Monitoring and Computing 15(7-8), 549-552.

[10] Fürstenau, N. (2016). Preface. In N. Fürstenau (Ed). Virtual and Remote Control Tower (p. xii). Cham (ZG): Springer International Publishing Switzerland.

[11] SAAB (2017). REMOTE TOWER REVOLUTIONISES AIR TRAFFIC MANAGEMENT. $S A A B$. Retrieved from: http://saabgroup.com/Media/stories/stories-listing/2017$\underline{02 / \text { remote-tower-revolutionises-air-traffic-management/ }}$

[12] Waller, M. J. (1997). Keeping the pins in the air: How work groups juggle multiple tasks. In M. M Beyerlein \& D. A. Johnson (Eds.), Advances in interdisciplinary studies of work teams (Vol. 4, pp. 217-247). Stamford, CT : JAI Press.

[13] Burak, L. (2012). Multitasking in the university classroom. International Journal for the scholarship of teaching and learning, 6(2), 8.

[14] Kahneman, D. (1973). Attention and effort (Vol. 1063). Englewood Cliffs, NJ: Prentice-Hall.

[15] Schumacher, E. H., Seymour, T. L., Glass, J. M., Fencsik, D. E., Lauber, E. J., Kieras, D. E., \& Meyer, D. E. (2001). Virtually perfect time sharing in dual-task performance: Uncorking the central cognitive bottleneck. Psychological science, 12(2), 101-108.

[16] Duncan, J., \& Mitchell, D. J. (2015). Training refines brain representations for multitasking. Proceedings of the National Academy of Sciences, 112(46), 14127-14128.

[17] Logan, G. D. (1988). Toward an instance theory of automatization. Psychological review, 95(4), 492.

[18] Loukopoulos, L. D., Dismukes, R. K., \& Barshi, I. (2009). The multitasking myth: Handling complexity in realworld operations. Routledge.

[19] Nijboer, M., Borst, J., van Rijn, H., \& Taatgen, N. (2014). Single-task fMRI overlap predicts concurrent multitasking interference. NeuroImage, 100, 60-74.

[20] Treisman, A., \& Davies, A. (1973). Divided attention between eye and ear. Attention and Performance $I V, S$. KORNBLUM (Editor). Academic Press, New York.

[21] Wickens, C. D., Boles, D., Tsang, P., \& Carswell, M. (1984). The limits of multiple resource theory in display formatting: Effects of task integration. ILLINOIS UNIV AT URBANA-CHAMPAIGN.

[22] Salvucci, D. D., \& Taatgen, N. A. (2008). Threaded cognition: An integrated theory of concurrent multitasking. Psychological review, 115(1), 101.

[23] Kallus, K. W., Van Damme, D., \& Dittmann, A. (1999). Integrated Task and Job Analysis of Air Traffic Controllers-Phase 2-Task Analysis of Enroute Controllers.

[24] SESAR Joint Undertaking. Remote Tower for Multiple Airports. Project funded by SESAR Joint Undertaking under the European Union's Horizon 2020 research and innovation programme under grant agreement No 730195 .

[25] Papenfuss, A., \& Friedrich, M. (2016). Head up only a design concept to enable multiple remote tower operations. In Digital Avionics Systems Conference (DASC), 2016 IEEE/AIAA 35th (pp. 1-10). IEEE.

[26] Cooper, G. E., \& Harper Jr, R. P. (1969). The use of pilot rating in the evaluation of aircraft handling qualities (No. AGARD-567). Advisory Group for aerospace research and development Neuilly-Sur-Seine (France).

[27] Kirwan, B., Evans, A., Donohoe, L., Kilner, A., Lamoureux, T., Atkinson, T., \& MacKendrick, H. (1997, June). Human factors in the ATM system design life cycle. In FAA/Eurocontrol ATM R\&D Seminar, Paris, France (pp. 16-20).

[28] SESAR Solution PJ.05.02 Validation Report (VALR) for V2 - Part I. (2018). To be published.

[29] Brookhuis, K. A., \& de Waard, D. (2010). Monitoring drivers' mental workload in driving simulators using physiological measures. Accident Analysis \& Prevention, 42(3), 898-903. 


\section{BIOGRAPHY}

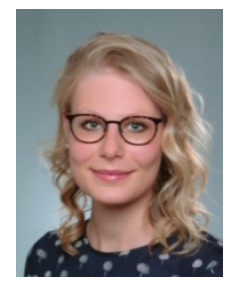

Maria Hagl received a Master's Degree in Psychology from Grenoble Alpes University. She is a research assistant and human factors expert at the German Aerospace Center, Institute of Flight Guidance. Since 2017 she works in the field of Remote Tower.

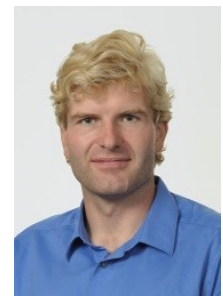

Maik Friedrich received a university degree for applied computer science (M. sc. 2008) at the Otto-Friedrich Universität in Bamberg, he started working as a full time researcher for the Human Factors department at the Institute of Flight Guidance, German Aerospace Center. He participated at the Remote Tower Projects RapTor and RAiCe and hat the responsible project management position for the SESAR 6.8.4 RTO.

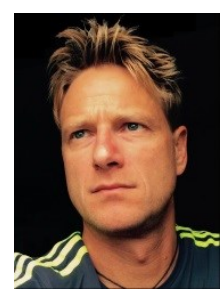

Jörn Jakobi received a diploma in Psychology (1999). Since 2000 he is as a human factors expert with DLR institute of flight guidance where he works in the domain of airport airside traffic management with the focus on A-SMGCS and Remote Tower concept operations and validation. Since 2010 he additionally works as a business developer for DLR with main focus on remote tower operations. In 2014 he became chairman of the EUROCAE WG100 "Remote \& Virtual Tower".

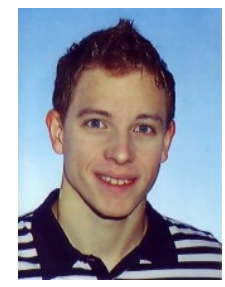

Sebastian Schier-Morgenthal studied Computer Science at the Technical University of Braunschweig. Since 2004 he works as a scientific expert for human in the loop simulations at the German Aerospace Centre (DLR). Currently he is responsible for the tower and airport management simulations at the Institute of Flight guidance.

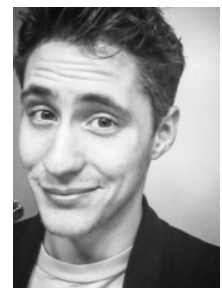

Christopher Stockdale is a Bachelor's student of Embry-Riddle Aeronautical University focusing in Human Factors Psychology as well as Space Operations and Policy. Christopher is currently an intern with the German Aerospace Center, Institute of Flight Guidance. 